LBNL-48814

\title{
Technical Design Aspects of Feasibility Study-IF
}

\author{
August 15, 2001 \\ M. S. Zisman \\ Center for Beam Physics \\ Accelerator \& Fusion Research Division \\ Lawrence Berkeley National Laboratory
}

Contribution to Neutrino Factory 2001 Workshop, May 24-30, 2001, Tsukuba, Japan To be published in Nuclear Instruments and Methods in Physics Research, Section A

\footnotetext{
"Supported by the U.S. Department of Energy, Division of High Energy Physics, under contract DE-AC0376 SF00098.
} 


\title{
Technical Design Aspects of Feasibility Study-II *
}

\author{
M. S. Zisman \\ Lawrence Berkeley National Laboratory, Berkeley, CA 94720 U.S.A.
}

\begin{abstract}
Feasibility Study-II examined a high-performance Neutrino Factory providing $1 \times$ $10^{20}$ neutrinos per year aimed at a long-baseline detector. The Study was sponsored jointly by BNL and the Neutrino Factory and Muon Collider Collaboration (MC) and is based on a $1 \mathrm{MW}$ proton driver operating at $24 \mathrm{GeV}$, i.e., an upgraded version of the AGS accelerator. Compared with the earlier FNAL-sponsored study (Feasibility Study-I), there is a sixfold improvement in performance. Here we describe details of the implementation of Study-II concepts and discuss their efficacy. Alternative approaches that will be pursued in follow-on R\&D activities are also described briefly.
\end{abstract}

Key words: Neutrino, Factory, Muon, Storage Ring

\section{Introduction}

Feasibility Study-II [1] is an outgrowth of the activities begun by FNAL and the MC (referred to herein as Study-I [2])to examine the ingredients of a Neutrino Factory as an integrated facility. Both Study-I and -II are site specific in that each has a few site-dependent aspects; otherwise, they are generic. In particular, Study-II used BNL site-specific proton driver specifications corresponding to an upgrade of the 24-GeV AGS complex and a BNL-specific layout of the storage ring, which is housed in an above-ground berm to avoid penetrating the local water table. Study-I used a new Fermilab booster to achieve its beam intensity and an underground storage ring. The primary substantive difference between the two studies is that Study-II aimed at a lower muon energy $(20 \mathrm{GeV})$, but higher intensity (for physics reach). Taken together, the

\footnotetext{
* Supported by the U.S. Department of Energy, Division of High Energy Physics, under contract DE-AC03-76SF00098.
} 
two Feasibility Studies show that a high-performance Neutrino Factory could easily be sited at either BNL or Fermilab.

The general approach used in Study-II was similar to that in Study-I. However, all designs were revisited with the goal of increasing the performance of the facility. In particular, a mercury-jet target was used as the baseline for StudyII and the phase rotation system was made non-distorting by using three induction linac units compared with only a single unit in Study-I. To optimize overall performance, the front-end systems (phase rotation, bunching, and cooling) were treated in an integrated fashion. This was one of the valuable lessons learned only at the end of Study-I.

\section{Technical Implementation}

\subsection{Target}

The baseline target used for Study-II is a mercury jet. It has the advantage of having a high $Z$, thus giving good pion yield with a $24-\mathrm{GeV}$ proton beam, and it can tolerate the high power deposited by a multi-MW incident proton beam better than a solid target could. Although the target material interacting with a given proton pulse will be completely dispersed, at a $\mathrm{Hg}$ jet velocity of 30 $\mathrm{m} / \mathrm{s}$ the jet is completely replenished during the $20 \mathrm{~ms}$ interval between proton beam pulses. A solid dump for the proton beam would likewise be expected to suffer mechanical degradation from the beam. To avoid this, we adopted a mercury pool for the beam dump. The beam dump thus serves as a reservoir for the $\mathrm{Hg}$, which is circulated from the pool to the jet orfice by a system of pumps along with heat exchangers. An artist's conception of the mercury circulation system is shown in Fig. 1.

Surrounding the target is a pair of nested solenoids that together produce a containment field of $20 \mathrm{~T}$. In Study-II, the inner magnet is a 6-T hollowconductor solenoid based on a MgO-insulated copper conductor. The outer magnet is a superconducting (SC) solenoid capable of providing $14 \mathrm{~T}$. The advantage of this approach is that the inner magnet is expected to have a much longer lifetime than the Bitter coil solenoid assumed for Study-I, but this comes at the expense of considerably higher operating-power requirements and poorer shielding efficacy for the surrounding SC magnet. To avoid difficulties with launching the $\mathrm{Hg}$ jet into a highly nonuniform field, we employ an iron plug to flatten the field profile and we locate the jet nozzle at the end of the plug, close to the maximum field point. 


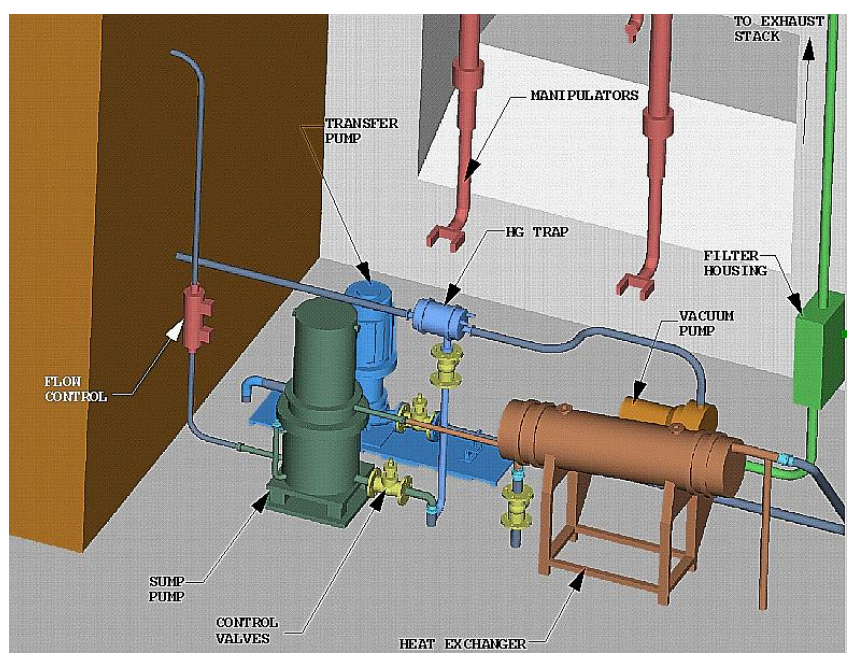

Fig. 1. Artist's concept of mercury target processing loop.

\subsection{Phase Rotation}

We perform phase rotation with three induction linac units $(100 \mathrm{~m}, 80 \mathrm{~m}$, and $80 \mathrm{~m}$, respectively) that provide the waveforms shown in Fig. 2. Each linac has internal SC solenoid focusing with a $0.5-\mathrm{m}$ period to contain the muon beam. The linac core size is similar to that of the DARHT accelerator [3], now under construction at LBNL. The choice of using three units rather than two units was made to avoid the use of a bipolar waveform that would be needed if IL2 and IL3 were implemented as a combined unit. The possibility of using a single core that is driven by two separate unipolar pulsers offers promise in saving costs and will be examined in the future.

The main technical challenge in the system proposed involves the effect on the induction linac core of the fringe field from the internal superconducting solenoid. The cost of the system is dependent on the inner radius of the core, so the closer the core can be brought to the solenoid the greater the savings. The

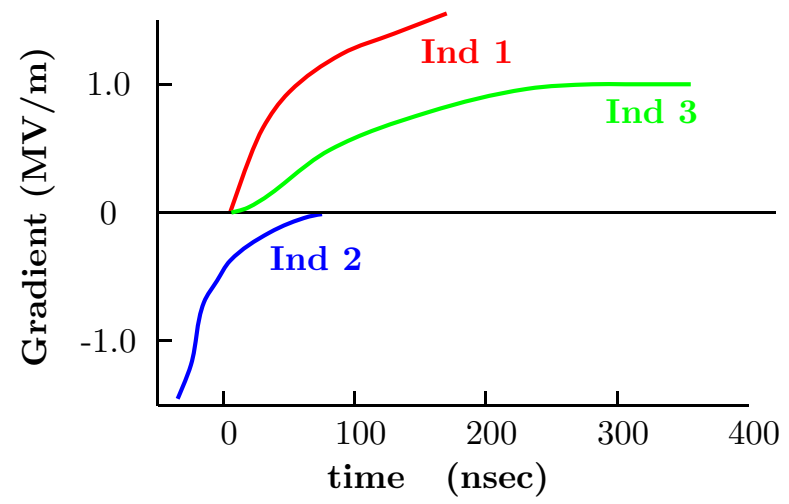

Fig. 2. Induction linac waveforms. Only that portion of the waveform seen by the muons is displayed. 


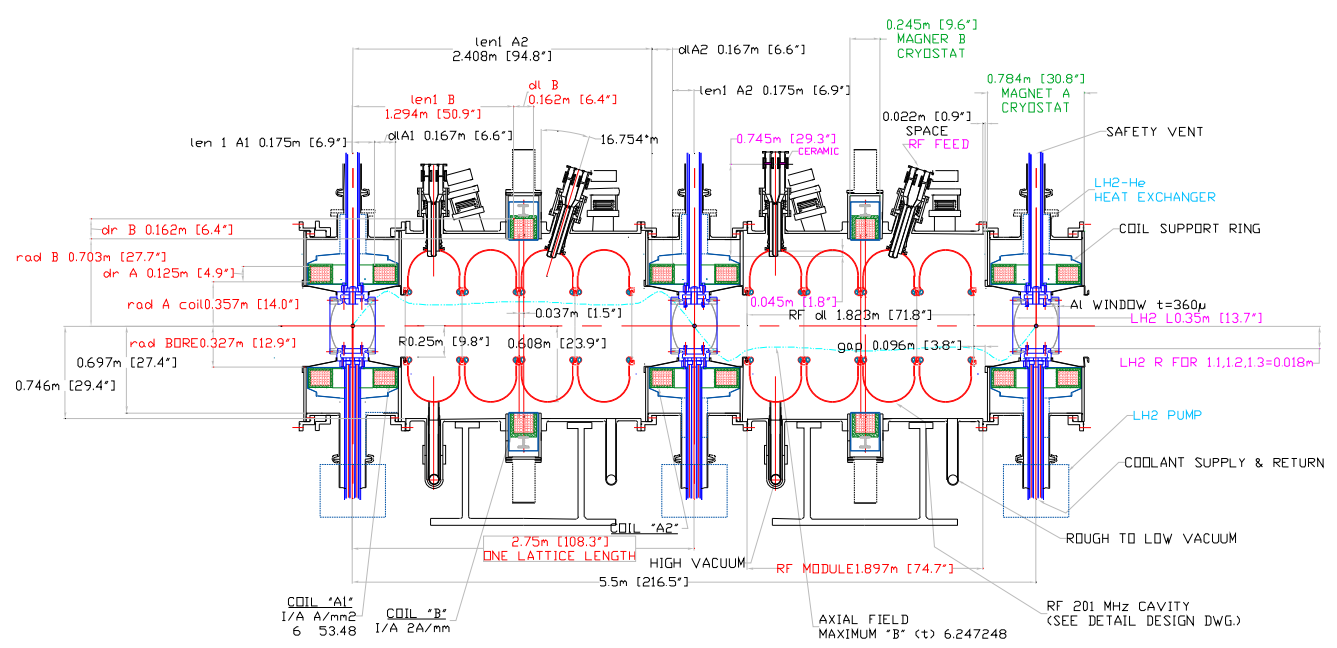

Fig. 3. Assembly drawing of SFOFO1 lattice showing rf cavities, solenoid coils, and absorbers.

present design is feasible, but $R \& D$ to see if it can be further cost optimized is planned.

\subsection{Buncher and Cooling Channel}

The beam that exits the phase rotation channel is a single long pulse, roughly $100 \mathrm{~ns}$ in duration. To reduce its transverse emittance, we need first to bunch it into 201-MHz pulses suitable for the cooling channel. This is done with a buncher section having the same magnetic lattice ("SFOFO") as the cooling channel and having rf cavities operating at either $201.25 \mathrm{MHz}$ or twice this frequency. The addition of a second rf harmonic helps improve the bunching efficiency by linearizing the rf waveform. The $201.25 \mathrm{MHz}$ buncher cavities are the same as the cavities used in Lattice 1 (2.75 m cell length), illustrated in Fig. 3.

The rf cavities illustrated in Fig. 3 are designed to have their apertures closed off with Be foils. The foils serve to increase the shunt impedance and on-axis accelerating field of the large-aperture cavities, reducing the power required to generate the specified gradient of $17 \mathrm{MV} / \mathrm{m}$. We assume stepped foils, with twice the thickness beyond the step radius $\left(r_{s}=\frac{2}{3} r_{\text {bore }}\right)$ as at the beam axis. The choice of Be is determined by its low $Z$ and acceptable electrical properties; its effectiveness is confirmed by our simulation codes. Even with the foils, we need about $5 \mathrm{MW}$ of rf power per cavity to generate a gradient of $17 \mathrm{MV} / \mathrm{m}$. We envision using a multibeam klystron to provide this power.

To provide the energy loss, we use $\mathrm{LH}_{2}$ absorbers contained between thin $\mathrm{Al}$ windows. These give rise to roughly $10 \mathrm{MeV}$ energy loss in each absorber for the central muon momentum of $200 \mathrm{MeV} / \mathrm{c}$. To optimize cooling, the lattice 


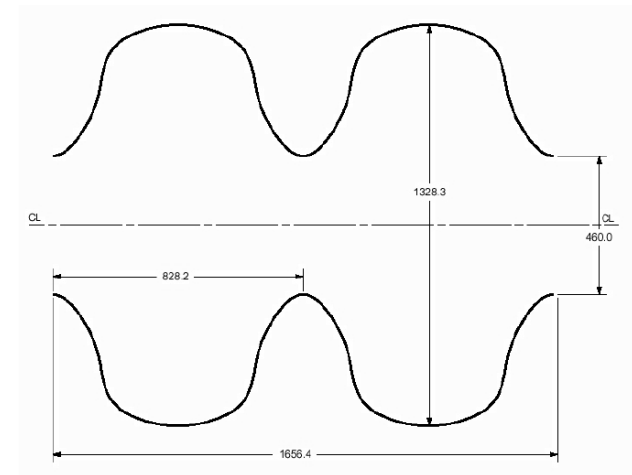

Fig. 4. Cavity shape for SCRF to give $17 \mathrm{MV} / \mathrm{m}$ gradient in linac and RLA.

period in the cooling channel is reduced from 2.75 to $1.65 \mathrm{~m}$ part way along the channel. In addition, the strength of the solenoidal field increases along the channel in such a way as to keep the divergence angle at the absorber roughly constant as the emittance decreases. Designs for the absorbers have been worked out [4]. Two approaches to heat removal are under study, based on internal or external heat exchangers. The parameter regime of interest, while difficult, is consistent with what has been achieved elsewhere with hydrogen targets.

The solenoid requirements are generally straightforward to achieve. The most challenging solenoids are the focusing coils at the $\mathrm{LH}_{2}$ absorbers in the short period lattice. As designed in Study-II, the magnetic forces are very high at this location due to the proximity of the coils and the high fields at the coil demand operation at $2 \mathrm{~K}$ to give sufficient margin. The next iteration of the design will increase the period length sufficiently to avoid this.

\subsection{Acceleration}

The rapid acceleration of the short-lived muons from the cooling channel momentum of $200 \mathrm{MeV} / \mathrm{c}$ to the final momentum of $20 \mathrm{GeV} / \mathrm{c}$ makes use of a "preacceleration" linac to bring the beam to $2.35 \mathrm{GeV}$, followed by a recirculating linear accelerator (RLA). Both the linac and the RLA are based on 201.25 MHz SCRF cavities [5]. (The power requirements make the use of NCRF unattractive for this portion of the facility.) A cavity shape has been developed, Fig. 4, that should permit attaining accelerating gradients of 17 $\mathrm{MV} / \mathrm{m}$. An example of a cryomodule for the RLA is shown in Fig. 5; the layout for the linac is similar except that the quadrupole triplet focusing is replaced by solenoidal focusing. In the linac, attention was paid to reducing the solenoid fringe field at the cavities to acceptable levels. Beam dynamics in the RLA has been studied and shown to be acceptable [6]. 


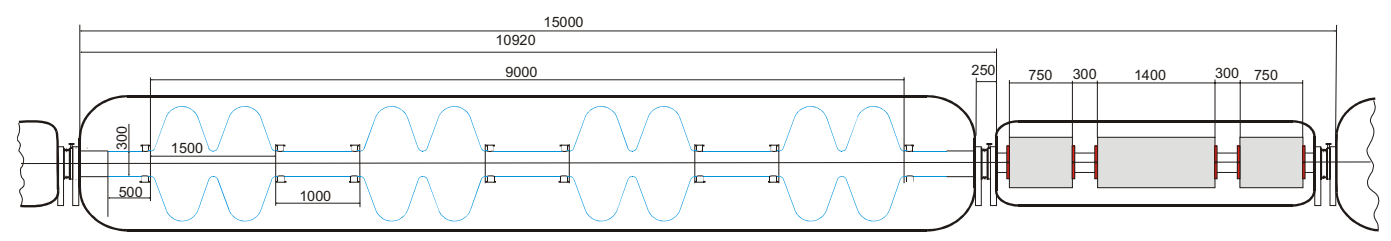

Fig. 5. Period of RLA lattice, showing rf cavities and quadrupole triplet.

\subsection{Storage Ring}

Design of a storage ring for a Neutrino Factory is reasonably straightforward [7]. The main design criterion is to have a long production straight section with low beam divergence. In practice, this is not hard, but the transition from the low beta function in the arcs to the high beta function in the straight section requires some space to achieve, and this matching region has beam properties unsuitable for the experiment. A workaround is possible, but it does come at a cost - the percentage of the ring circumference available for neutrino beam production for the long baseline experiment is reduced from about $35 \%$ to $27 \%$ by the matching section.

For Study-II, the BNL site dictated a second constraint for the storage ring design. The need to avoid penetrating the local water table led to housing the ring in an above-ground berm. This places a premium on a compact ring and led to the design of a skew quadrupole lattice as described in Ref. [7]. A more conventional approach could also be used, albeit with a small penalty on the overall ring circumference.

\section{Alternative Approaches}

\subsection{Rotating Band Target}

While it has not been studied as carefully as the baseline design, the alternative of a rotating inconel-band target has also been considered [8]. The yield expected from such a target is comparable to that of $\mathrm{Hg}$. In this case, the potential material handling issues with liquid mercury are replaced by the mechanical issues associated with a large, rapidly rotating metal band that must penetrate into the target solenoid magnetic field. 


\subsection{RF Phase Rotation}

An interesting possibility, raised by Neuffer [9], is to use a series of rf cavities to perform the phase rotation and bunching. This approach requires considerably more rf hardware, at various frequencies, but it eliminates the need for induction linacs entirely. Detailed simulations are required to assess the efficiency of the approach, but initial exploration looks promising. Simulations from target through cooling channel will be needed to validate the approach, along with a cost estimate for the required hardware.

\subsection{Fixed-Field Alternating Gradient Acceleration}

Another area that must be explored in more detail is the use of Fixed-Field Alternating Gradient (FFAG) acceleration. There is considerable work on this subject going on in Japan, but the idea we wish to examine involves an FFAG ring with localized high-frequency rf. In effect, we consider an RLA in which the multiple arcs are combined into an FFAG structure capable of accommodating the entire energy swing of the RLA [10]. Some design work along these lines has already been done, but further optimization and a cost estimate are needed.

\subsection{Conventional Compact Storage Ring}

To compare with the work done in Study-II on compact skew quadrupole lattices, a corresponding effort is needed on conventional approaches. Initial efforts have already shown [11] that both combined-function and separatedfunction lattices based on conventional magnets can give a circumference comparable to that of the skew quadrupole lattice [7] used in Study-II. The disadvantage of the conventional approach is that a liner is needed to shield the magnets from the muon decay products. In the Study-II approach, the median plane contains no coils, so a warm-iron support absorbs the energy. Comparisons are needed on both magnet quality (which affects the dynamic aperture of the ring), and magnet costs.

\section{Summary}

In this paper we have briefly presented the technical design aspects of the Feasibility Study-II Neutrino Factory [1]. For all subsystems, we have identified 
technical approaches that will provide the required performance, assuming that the component specifications can be met. The design studied would be compatible with siting either at BNL or at FNAL. An R\&D plan to address the component performance issues has been formulated and is presently under way. Alternative design options are also under study to explore possibilities for performance enhancements and/or cost reductions.

\section{Acknowledgments}

I am grateful for the support of my Study-II co-leaders, S. Ozaki and R. Palmer during the course of the Study. The work presented here is due to the efforts of the many participants in the Study, and especially to the technical editors of the various sections and the report editor, J. Gallardo, who pulled the work together in a coherent fashion. Support from the management of BNL was also key to completing the work successfully. Lastly, the enthusiasm and effort of the MC, and especially its Spokesperson, A. Sessler, in carrying out this work were critical and are greatly appreciated. The help of J. S. Berg in the preparation of the final manuscript was invaluable.

\section{References}

[1] "Feasibility Study-II of a Muon-Based Neutrino Source," S. Ozaki, R. Palmer, M. S. Zisman, J. Gallardo, eds., BNL-52623, June, 2001. http://www.cap.bnl.gov/mumu/studyii/final_draft/The-Report.pdf

[2] "Feasibility Study of a Neutrino Source Based on a Muon Storage Ring," N. Holtkamp and D. Finley, eds., Fermilab-Pub-00/108-E (2000), http://www.fnal.gov/projects/muon_collider/nu-factory/nu-factory.html

[3] M. J. Burns et al., "DARHT Accelerators Update and Plans for Initial Operation," in Proc. 1999 Particle Accelerator Conf., p. 617.

[4] D. M. Kaplan et al., "Progress in Energy Absorber R\&D 2: Windows," in Proc. of 2001 Particle Accelerator Conf., Chicago, to be published.

[5] H. Padamsee, "Superconducting RF - New Directions," in Proc. of 2001 Particle Accelerator Conf., Chicago, to be published.

[6] J. Delayen et al., "Issues in Acceleration of a Muon Beam for a Neutrino Factory" in Proc. of 2001 Particle Accelerator Conf., Chicago, to be published.

[7] B. Parker, "Skew Quadrupole Focusing Lattices and Applications," in Proc. of 2001 Particle Accelerator Conf., Chicago, to be published. 
[8] B. J. King, "A Rotating Inconel Band Target for Pion Production at a Neutrino Factory, Using Study-II Parameters," MC Note-199, http://wwwmucool.fnal.gov/mcnotes/muc0199.pdf.

[9] D. Neuffer, "High-Frequency Buncher and $\phi-\delta E$ Rotation for the $\mu^{+}-\mu^{-}$ Source," MC Note-181, http://www-mucool.fnal.gov/mcnotes/muc0181.pdf.

[10] C. Johnstone, W. Wan, and A. Garren, "Fixed Field Circular Accelerator Designs," in Proc. of 1999 Particle Accelerator Conference, p. 3068, 1999.

[11] C. Johnstone and A. Garren, "Racetrack Shaped Muon Storage Rings for a Neutrino Factory," in Proc. of 2001 Particle Accelerator Conf., Chicago, to be published. 